\title{
Distal Tibia Delayed Union with Fibular Shortening Causing Syndesmotic Instability and Anterolateral Impingement
}

\author{
Austin J Cantrell ${ }^{1}$, Joshua A Wilson ${ }^{2}$, Amgad Haleem ${ }^{3}$, Seth Boydstun ${ }^{4}$
}

\begin{abstract}
Debate continues in the current orthopedic literature regarding advantages of performing concurrent fibular stabilization in combined distal tibial and fibular fractures. In this case report, we present the correction of tibial malalignment, fibular fixation, and syndesmotic restoration in a 21-year-old male after a high-energy injury where he sustained a fracture of the tibia and fibula at the same level that was initially managed with tibial nailing alone. After initially having delayed union, valgus malalignment and resultant syndesmotic pain and instability following the index surgery, he underwent revision surgery resulting in favorable recovery with cessation of his pain and correction of his malalignment. Keywords: Distal tibia and fibula fracture, Intramedullary nail, Malalignment.

Journal of Orthopedics and Joint Surgery (2019): 10.5005/jp-journals-10079-1009
\end{abstract}

\section{INTRODUCTION}

There is a debate throughout the literature about the optimum treatment of concomitant distal tibia and fibula fractures. The debate primarily centers around whether to perform a tibia intramedullary nail (IMN) alone or to concurrently perform an open reduction internal fixation of the fibula. The proponents of the tibialfixation-only method argue that the restoration of tibial length and that alignment restores fibular length with no long-term functional dysfunction. ${ }^{1}$ The proponents of combined fixation note decreased the rate of malalignment. ${ }^{2}$ It is our belief that the initial restoration of fibular length may help to prevent sequela of syndesmotic pain and instability. In this case report, we present the correction of tibial malalignment, fibular fixation, and syndesmotic restoration after a high-energy injury fracture of the tibia and fibula at the same level that was initially managed with tibial nailing alone. Our aim was to highlight the potential sequela of chronic syndesmotic pain and subsequent instability that may occur in these injuries and provide a technique for revision should an inferior outcome result following tibial nailing alone.

\section{Case Description}

A 21-year-old male presented to our trauma department with a high-energy fracture to the lower limb after being struck while riding his motorcycle. Patient denied any numbness or weakness. Past medical history was unremarkable. Physical examination revealed a $6 \mathrm{~cm}$ laceration at the anteromedial aspect of the leg with exposed muscle, tendon, and bone. X-ray revealed fractures of the tibia and fibula at the same level (Fig. 1).

The patient underwent irrigation and debridement of the open wound and an IMN to his tibia. Two proximal and two distal interlocking screws were used to secure the nail in place. The fibula fracture was not plated, and the syndesmosis was felt to be uninvolved (Fig. 2). The surgery was uneventful.

The patient returned for 6-week follow-up. Radiographs at that time showed a lack of bone healing in both tibia and fibula, as well as a new-onset valgus angulation of the lower limb nearing $5^{\circ}$ (Fig. 3). Fibular length was shorter than normal, and the distal fibular "dime sign" was disrupted. At follow-up one month later (11 weeks
${ }^{1-4}$ Department of Orthopedic Surgery and Rehabilitation, University of Oklahoma Health Sciences Center, Oklahoma City, Oklahoma, United States of America

Corresponding Author: Austin J Cantrell, Department of Orthopedic Surgery and Rehabilitation, University of Oklahoma Health Sciences Center, Oklahoma City, Oklahoma, United States of America, Phone: +1 405-320-4048, e-mail: austin-cantrell@ouhsc.edu

How to cite this article: Cantrell AJ, Wilson JA, Haleem A, et al. Distal Tibia Delayed Union with Fibular Shortening Causing Syndesmotic Instability and Anterolateral Impingement. J Orth Joint Surg 2019;1(1):31-34.

Source of support: Nil

Conflict of interest: None

postoperative), radiographs continued to show delayed healing of the tibia and fibula and an even greater valgus angulation of the lower limb approaching $7^{\circ}$ (Fig. 4). Fibular length remained short. The patient reported increasing pain in his ankle, specifically his anterolateral ankle around the syndesmosis. Examination revealed tenderness at the tibial fracture site with a clinical valgus of $7^{\circ}$
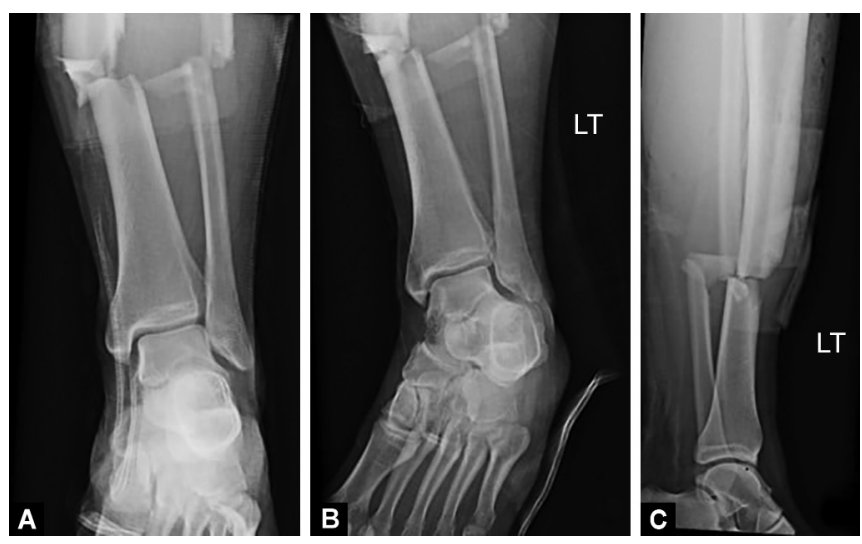

Figs 1 A to C: Initial injury films: (A) AP of ankle; (B) Mortise of ankle; (C) Lateral of tibia 

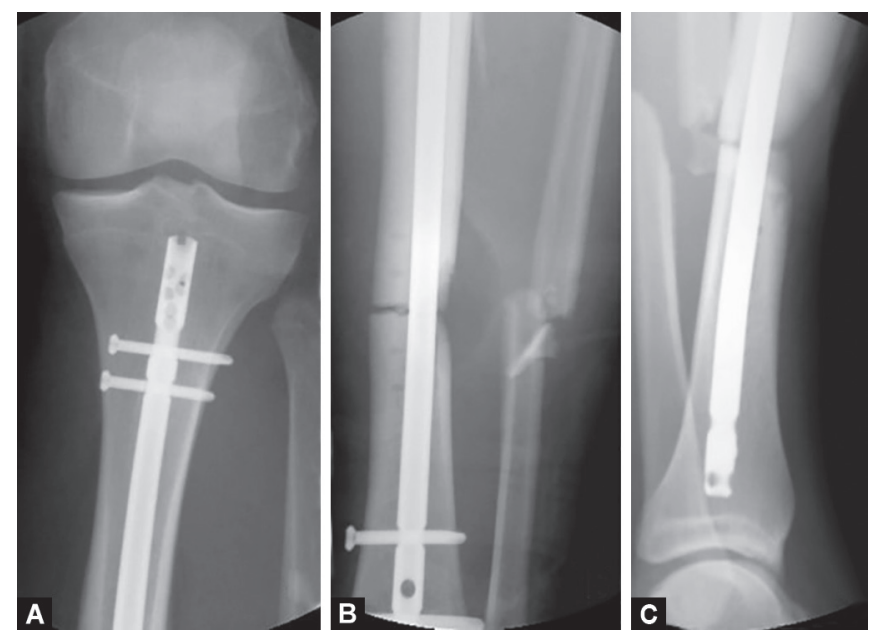

Figs $2 \mathrm{~A}$ to $\mathrm{C}$ : Intraoperative radiographs after initial operation: (A) AP of proximal interlock screws; (B) AP of fracture site; (C) Lateral of fracture site
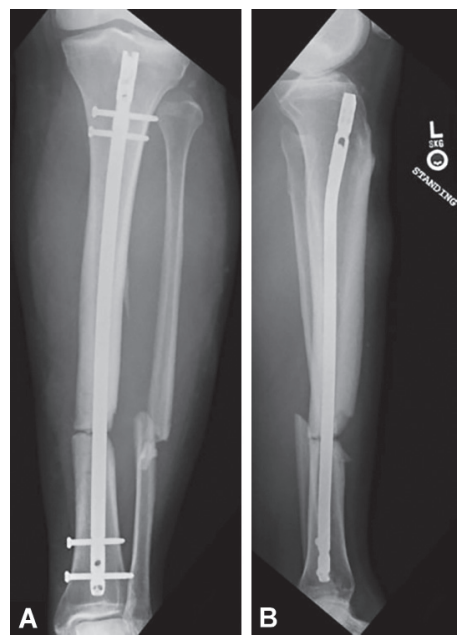

Figs 4A and B: Radiographs 11 weeks postoperatively following the initial surgery showing valgus deformity approaching $7^{\circ}$. Note the lack of callus formation about the tibia or fibula fractures: (A) Lateral of tibia; (B) AP of tibia

on standing, syndesmotic instability with tenderness, a positive squeeze, and external rotation stress tests.

The patient returned the following month (15 weeks postoperative), and the radiograph results remained the same (Fig. 5). At this time, the patient was referred to the lower extremity complex reconstruction service, where further surgical intervention was deemed necessary to address the increasing valgus, delayed union with pain, fibular shortening, and resultant syndesmotic instability/disruption. It was deemed reasonable at that time to intervene with corrective surgery to address all aforementioned pathologies.

The second surgery was performed 22 weeks status post his initial surgery. First, the two distal interlocking screws were removed to dynamize the nail. Additionally, two Poller blocking screws were placed at the distal end of the tibia, lateral to the nail after correcting the valgus deformity following the principles of placement of Poller screws in the concavity of the anticipated deformity. Furthermore, the fibula underwent lengthening with a step cut osteotomy and open reduction internal fixation using
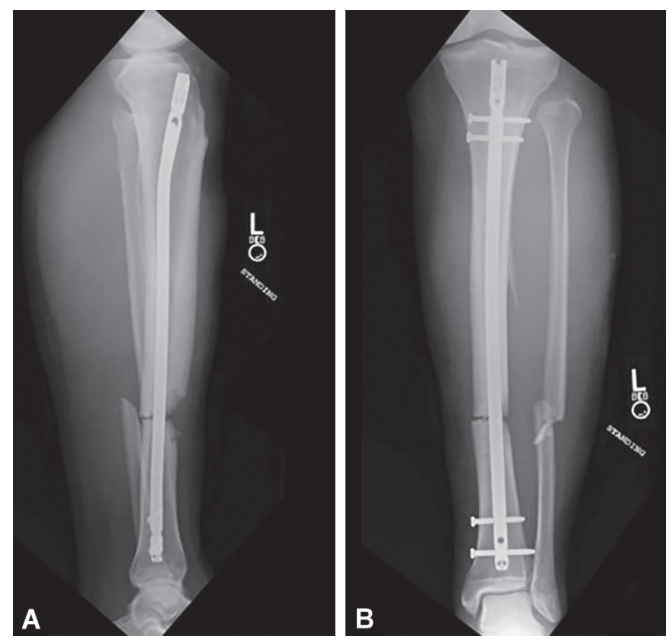

Figs $3 A$ and B: Radiographs 6 weeks postoperatively following the initial surgery showing slight valgus deformity of approximately $5^{\circ}$. Note the disruption of the distal fibular dime sign: (A) Lateral of tibia; (B) AP of tibia
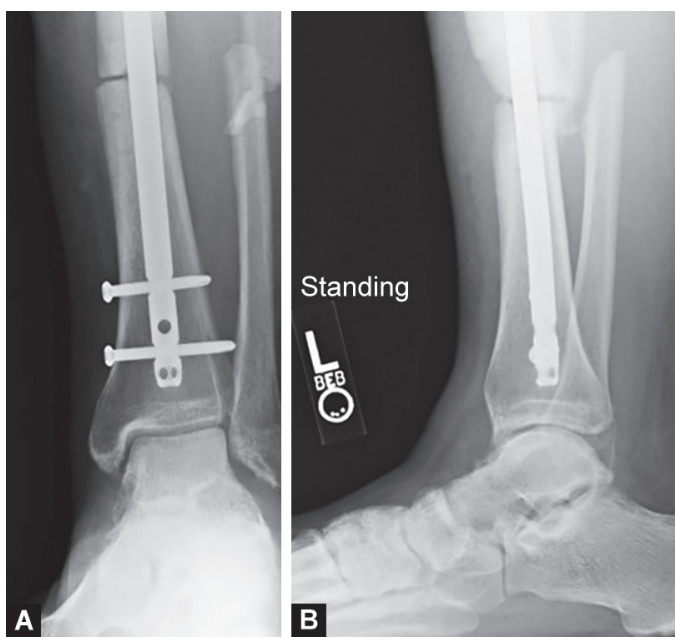

Figs 5A and B: Radiographs 15 weeks postoperatively following the initial surgery. Note the lack of callus formation about the tibia or fibula fractures: (A) AP of ankle; (B) Lateral of ankle

a 1/3 tubular plate and $3.5 \mathrm{~mm}$ nonlocking cortical screws. Step cut osteotomy was chosen to allow for appropriate lengthening without significantly medially displacing the distal fibular fragment and hindering plate positioning in case an oblique osteotomy was used.

Finally, after restoring fibular length, knotless syndesmotic tight ropes (Arthrex, Naples, FL, USA) were used to address the syndesmotic pain and instability. Two tight ropes were placed (Fig. 6). The surgery was uneventful.

The patient returned for 6-week follow-up from the second surgery. At that time, the radiographs showed interval healing of the tibia and fibula. The patient reported that syndesmotic pain had decreased. Also, the patient's fibula length was more anatomic, and the distal fibular dime sign had been restored. The patient's status was followed for 41 weeks postoperatively. During the follow-up period, bone healing continued, syndesmotic pain had decreased, and valgus malalignment did not exceed $2^{\circ}$ (Fig. 7). The patient was completely asymptomatic and returned to his daily activities without incapacitation. 


\section{Discussion}

Morin et al. performed a mechanical testing to determine the benefits of plating the fibula on stabilization of the lower limb. They found the only significant difference seen was in limiting axial rotation during torsional strain. ${ }^{3}$ This was confirmed by a clinical study that showed that torsional injuries to the tibia and fibula required both tibia and fibula fixation to achieve a proper alignment. ${ }^{4}$ Another clinical study stated that a concurrent fibula fixation maintained alignment at a slightly higher rate than IMN of the tibia alone, and significantly improved the ability to maintain fracture fixation past 12 weeks. ${ }^{2}$ However, a recent study concluded that fixing a distal tibia and fibula fracture with an IMN of the tibia alone was sufficient in providing a low malalignment and malunion rate and stated that fibula fixation is not a requirement. ${ }^{1}$ As demonstrated by this case, an IMN of the tibia alone did not provide the proper union and alignment desired.

When considering how to address the delayed or nonunion in tibial shaft fractures, dynamization of the nail is typically a first-line therapy once other causes of delayed union have been ruled out

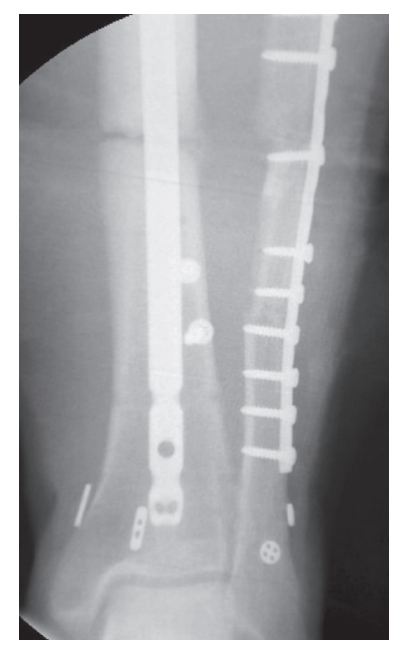

Fig. 6: Intraoperative radiographs of the second surgery showing removal of the distal interlock screws, placement of blocking screws, syndesmotic fixation, and open reduction internal fixation of the fibular fracture (infection and metabolic deficiency). Freeing the distal portion of the tibia allows for greater contact area of the two portions of bone and greater osteogenesis, and it is associated with low cost and morbidity. ${ }^{5}$ After dynamization, the use of Poller blocking screws has proven successful in correcting and preventing malalignment and increasing the stability of the nail. ${ }^{6}$

In 1999, Krettek et al. described the first use of Poller blocking screws for the prevention of malalignment of proximal and distal third fractures of tibia during the process of IMN.,8 The acceptable malalignment of a tibia shaft is less than $5^{\circ}$ of varus-valgus angulation, $10^{\circ}$ of anteroposterior (AP) angulation, $10^{\circ}$ of rotation, and $15 \mathrm{~mm}$ of shortening. ${ }^{7}$ Blocking screws functionally reduce the width of metaphysis intramedullary diameter and are usually applied from anterior to posterior to correct coronal plane malalignment. ${ }^{7}$ Krettek et al. recommend placing one screw proximally and one distally on the concave side of the displacement., 9,10 According to Stedtfeld et al., the key to preventing malalignment is three-point fixation within the intramedullary canal. This can be achieved with the use of a single transmedullary blocking screw if there are two other adequate points of fixation. ${ }^{10}$ When the choice is made to not use blocking screws and malalignment of a small degree is accepted, degenerative changes of adjacent joints may follow. Van der Schoot et al. recorded a 15-year follow-up of 88 patients with fractures of lower leg in which $49 \%$ of the patients healed with malalignment of at least $5^{\circ} ., 11$ They showed that there was significantly more arthritis in the knee and ankle adjacent to the fracture than in the same joints of the contralateral uninjured leg. ${ }^{7,11}$ Puno et al. followed 28 tibia fractures with malalignment and showed that poorer clinical results were associated with ankle malalignment. ${ }^{7,12}$

Syndesmosis injury is also specifically evaluated for when the ankle fracture is isolated or if the fracture involves the distal third of the fibula. Commonly employed methods for evaluation of the syndesmosis are the external rotation stress test and the cotton test. The external rotation stress test, as the name implies, involves placing an externally directed force to the foot and evaluating for syndesmotic widening. The cotton test involves clamping the fibula with a towel clamp, and pulling laterally while taking an AP radiograph to determine whether there is any widening at the tibiofibular clear space. Both of these tests, however, generally require a fixation of the fibula prior to being
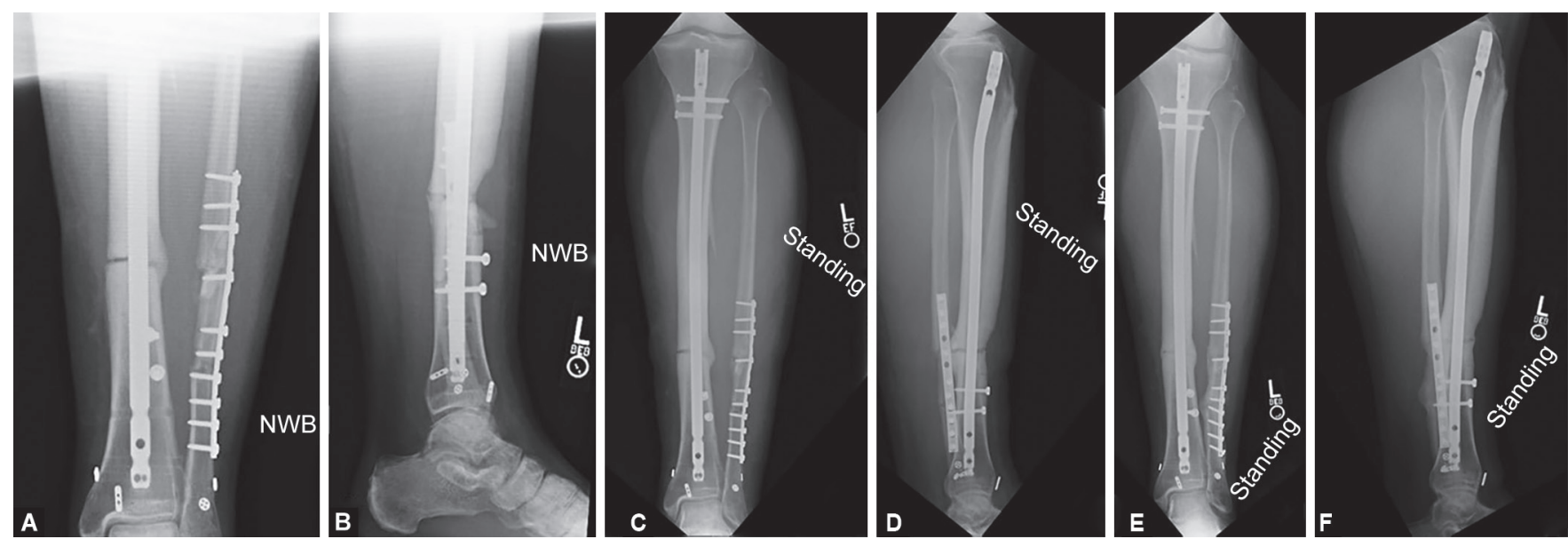

Figs 7A to F: Postoperative radiographs following second surgery showing healing of the tibia and fibular fractures with improved alignment of the tibia: (A and B) AP and lateral radiographs of tibia at 6 weeks following the second surgery; (C and D) AP and lateral radiographs of tibia at 15 weeks following the second surgery; (E and F) Final AP and lateral radiographs of tibia 10 months following the second surgery 
performed. Traditionally, Weber C fibula fractures have been thought to have a high incidence of syndesmosis injury. Weening and Bhandari showed that in ankle fractures where the syndesmosis was determined to be injured, $70 \%$ were associated with a Weber C fibula fracture. ${ }^{13}$ In their study, $16 \%$ of syndesmoses were not reduced. This was important as they stated the only modifiable risk factor of functional outcome after ankle fractures was reduction of the syndesmosis injury $(p=0.4) .^{13}$

The entire length of the fibula is required to maintain ankle stability. ${ }^{14}$ In our patient, we believe that the malalignment of the tibia and shortening of the fibula resulted in syndesmotic pain, attenuation, and ultimately instability. Restoring fibular length restored the syndesmotic anatomy, which, in turn, eliminated our patient's persistent pain. Given the revision setting, we chose to supplement or syndesmotic fixation utilizing the commercially available syndesmotic tight rope. We prefer tight rope to syndesmotic screws because the tight rope does not break and does not have to be removed with a subsequent surgery. The tight rope has also shown to decrease time to weight-bearing when compared to screw fixation. ${ }^{15}$

We acknowledge that the decision to plate the fibula along with IMN of the tibia increases surgery time and cost. However, if subsequent fibula fixation is required after an unsuccessful IMN of the tibia, the cost and time to the patient is more burdensome than doing one initial surgery accomplishing both. Additionally, this case highlights the importance of attention to ankle anatomy detail during surgical nailing of distal tibial fractures if a decision is made not to fix the fibula. We emphasize ensuring fibular length is restored to avoid valgus malalignment of the tibial fracture and future syndesmotic pain and instability. In addition, it is crucial to evaluate the ankle syndesmosis intraoperatively in order to make a decision for fibular/syndesmotic stabilization.

\section{Conclusion}

In conclusion, when presented with a patient with distal third tibia and fibula fractures, extra time should be taken to consider assessing fibular length and syndesmotic stability, with consideration of plating the fibula in conjunction with IMN of the tibia. The consideration of placement of Poller screws to prevent valgus malalignment and further fibular shortening is critical. In addition, initial plating of the fibula could limit potential malunion, malalignment, fibular shortening, and subsequent syndesmotic pain. We acknowledge that time and cost should be considered when debating this treatment but performing both initially has the potential benefits of improved patient outcome and decreased need for further procedures. This case highlights potential sequela of tibia only fixation, especially in situations with concern for syndesmotic injury, and provides a technique for addressing these issues should they occur.

\section{Ethical Standards}

Ethical Standards were met by not identifying the subject of the case report.

\section{References}

1. De Giacomo AF, Tornetta PIII. Alignment after intramedullary nailing of distal tibia fractures without fibula fixation. J Orthop Trauma 2016;30(10):561-567. DOI: 10.1097/bot.0000000000000637.

2. Egol KA, Weisz R, Hiebert R, et al. Does fibular plating improve alignment after intramedullary nailing of distal Metaphyseal tibia fractures? J Orthop Trauma 2006;20(2):94-103. DOI: 10.1016/s02761092(08)70090-7.

3. Morin PM, Reindl R, Harvey EJ, et al. Fibular fixation as an adjuvant to tibial intramedullary nailing in the treatment of combined distal third tibia and fibula fractures: a biomechanical investigation. Can J Surg 2008;51(1):45-50.

4. Bonnevialle $P$, Lafosse J-M, Pidhorz $L$, et al. Distal leg fractures: how critical is the fibular fracture and its fixation? Orthop Traumatol Surg Res 2010;96(6):667-673. DOI: 10.1016/j.otsr.2010.07.002.

5. Vaughn J, Gotha H, Cohen E, et al. Nail dynamization for delayed union and nonunion in femur and tibia fractures. Orthopedics 2016;39:e1117-e1123. DOI: 10.3928/01477447-20160819-01.

6. Dodd L, Jackson M, Varma R. Poller blocking screws and intramedullary nailing in tibial malunion. Ann R Coll Surg Engl 2007;89(8):816-818. DOI: 10.1308/rcsann.2007.89.8.816b.

7. Sengodan MM, Vaidyanathan S, Karunanandaganapathy $S$, et al. Distal tibial metaphyseal fractures: does blocking screw extend the indication of intramedullary nailing? ISRN Orthop 2014;2014:542623.

8. Krettek C, Miclau T, Schandelmaier P, et al. The mechanical effect of blocking screws ("Poller screws") in stabilizing tibia fractures with short proximal or distal fragments after insertion of small-diameter intramedullary nails. J Orthop Trauma 1999;13(8):550-553. DOI: 10.1097/00005131-199911000-00006.

9. Krettek C, Stephan C, Schandelmaier P, et al. The use of Poller screws as blocking screws in stabilising tibial fractures treated with small diameter intramedullary nails. J Bone Joint Surg Br 1999;81(6): 963-968. DOI: 10.1302/0301-620x.81b6.10000.

10. Stedtfeld HW, Mittlmeier T, Landgraf $\mathrm{P}$, et al. The logic and clinical applications of blocking screws. J Bone Joint Surg Am 2004; 86-A(Suppl 2):17-25. DOI: 10.2106/00004623-200412002-00004.

11. Van der Schoot DKE, Den Outer AJ, Bode PJ, et al. Degenerative changes at the knee and ankle related to malunion of tibial fractures. 15-year follow-up of 88 patients. J Bone Joint Surg 1996;78(5):722-725.

12. Puno RM, Vaughan JJ, Stetten ML, et al. Long-term effects of tibial angular malunion on the knee and ankle joints. J Orthop Trauma 1991;5(3):247-254. DOI: 10.1097/00005131-199109000-00001.

13. Weening B, Bhandari M. Predictors of functional outcome following transsyndesmotic screw fixation of ankle fractures. J Orthop Trauma 2005;19(2):102-108. DOI: 10.1097/00005131-200502000-00006.

14. Uchiyama E, Suzuki D, Kura $H$, et al. Distal fibular length needed for ankle stability. Foot Ankle Int 2006;27(3):185-189. DOI: 10.1177/107110070602700306

15. Porter DA, Jaggers RR, Barnes AF, et al. Optimal management of ankle syndesmosis injuries. Open Access J Sports Med 2014;5:173-182. DOI: 10.2147/OAJSM.S41564. 